Apidologie, 1977, 8 (4), 427-436.

\title{
EINIGE FAKTOREN, WELCHE DIE HONIGTAUZUSAMMENSETZUNG NATÜRLICH WIE AUCH HOLIDISCH ERNÄHRTER APHIDENLARVEN BEEINFLUSSEN
}

\author{
Quelques facteurs qui influencent la composition chimique \\ du miellat des larves d'aphidiens \\ nourries sur milieux naturel et holidique
}

\author{
Rolf HERTEL* und Hartwig KUNKEL \\ Institut für angewandte Zoologie \\ der Universität Bonn \\ An der Immenburg 1, 5300 Bonn, B.R.D.
}

\begin{abstract}
SUMMARY
SOME FACTORS INFLUENCING THE COMPOSITION OF HONEYDEW OF NATURALly AND holidicly FED APHid LARVAE
\end{abstract}

We compared honeydew of holidicly and plant fed larvae of the aphid Myzus persicae (Sulz.) by analysing the content of amino acids and carbohydrates (aldo- and ketohexoses). Working under standardized conditions, the content of these honeydew-compounds differs in respect of 1) the time of sampling for analysis, 2) the age of the animals and 3) the morphal stage of the aphids (apteriform-alatiform). Another factor, which influences the honeydew is the variable concentration of amino acids and carbohydrates in the holidic diet or the plant (changes in phloem sap content, changing of water supply).

\section{ZUSAMMENFASSUNG}

Wir analysierten die Zusammensetzung des Honigtaus von holidisch und natürlich ernährten Aphidenlarven hinsichtlich ihres Aminosäuren- und Kohlenhydratgehaltes. Unter standardisierten Bedingungen hängt die Zusammensetzung des Honigtaus von der Tageszeit der

* Gegenwärtige Adresse : Physiologisches Institut der Universität Würzburg, Röntgenring 9 , $\mathrm{D}-87$ Würzburg. 
Probennahme für die Analyse, vom Alter der Tiere sowie von der Zusammensetzung der Aphidenkolonie (anteilmäBiges Auftreten beider Morphen) ab. Daneben ist als weiterer Faktor das Nähr=toffangebot zu nennen, das unter natürlichen Bedingungen durch die Art und den Versorgungszustand der Wirtspflanze charakterisicrt ist.

\section{1. - EINLEITUNG}

In der Vergangenheit haben mehrere Autoren den Aphidenkot, auch Honigtau (H'T) genannt, untersucht. Dabei stellte Boussingault (1872) als erster fest, daß der Zuckergehalt seiner Proben, an verschiedenen Tagen gesammelt, eine unterschiedliche Zusammensetzung aufweist. Dieser Beobachtung ist in der folgenden Zeit wenig Aufmerksamkeit geschenkt worden : alle Autoren (Übersicht bei HerTeL, 1974b), die den HT untersuchten, haben ihn in längeren Zeiträumen gesammelt und dann analysiert, wobei sie keine weiteren biotischen oder abiotischen Angaben, die die Sammelzeit betreffen, machten. Hierzu Daten vorzulegen ist Anliegen unserer Arbeit.

\section{2. - MATERIAL UND METHODEN}

Seit Einführung von künstlicher Ernährung bei Aphiden (MrtTLER and DadD, 1962) und ihrer Optimierung für unser Versuchstier (zuletzt HenTes, 1974 a) kann der Einfluß der W irtspflanze, der sich z.T. unkontrolliert verändert, ausgeschaltet werden. Bei der so erhaltenen Ernährungslage zeigen Änderungen in der HT-Zusammensetzung unmittelbar Reaktionen des Tieres auf die gewählten Versuchsbedingungen. Wird der HT während längerer Zeit bei sonst gleichen Experimentalbedingungen von Aphiden gesammelt, die an Pflanzen parasitieren, wird seine Zusammensetzung von beiden Organismen bestimmt. Durch Vergleich der Analysen an beiden Nahrungsquellen können die einzelnen Faktoren getrennt dargestellt und bewertet werden.

Als Versuchstiere dienten Larven der Grünen Pfirsichblattlaus Myzus persicae (Sulz.), deren Haltungsbedingungen bereits beschrieben sind (HERTEL, 1971). Experimentiert wurde bei $20^{\circ} \mathrm{C}, 65 \%$ RF und Wechsellicht mit einer Scotophase von 8 Stunden.

Hertel (1971) hat ausführlich eine Methode beschrieben, HT von altersmäßig exakt definierten Aphidenkolonien zu erhalten. Zur Analyse der mengenmäßig wichtigsten Inhaltsstoffe des HT, der Aminosäuren (AS) und Kohlenhydrate (KH), wurden je Probe $50-100 \mathrm{Kot}$ tropfen gesammelt und mit $1 \mathrm{ml}$ bidest. $\mathrm{H}_{2} \mathrm{O}$ aufgenommen. Von dieser Lösung wurden 6 Einzelproben à $100 \mu \mathrm{l}$ für je drei KH- bzw. AS-Nachweise eatnommen. Als KH wurden Aldound Ketohexosen nebeneinander in der gleichen Probe mit Anthron (VoN Handes, 1967) bestimmt. Zur Analyse von ninhydrinpositiven Substanzen, in überwiegender Mehrheit AS, wurde die von uns modifizierte Methode von Rosen (1957) sowie eine Mikro-Kjeldahl-Methode mit anschließendem Harnstoffarbtest (s. a. HERTEL, 1971) benutzt; in diesem Fall verwandten wir auf Abbildungen als Index Stickstoff mit dem Symbol ' $N$ '.

Da der HT-Tropfen schon bei seinem Erscheinen am Anus der Aphide Wasser verdampft (in 30 sec. $30 \%$, KuNkel und Hertel, 1976) und damit erheblichen Volumen-und Konzentrationsänderungen unterworfen ist, haben wir den AS-Gehalt auf den HK-Gehalt in der gleichen Probe bezogen und diesen relativierenden Quotienten angegeben. Dort, wo das nicht sinnvoll erschien, haben wir den Gehalt in $\mu g$ pro $10 \mathrm{HT}$-Tropfen verwandt. Wir wählten diese ( 10-Tropfen-Einheit " als Erinnerung daran, daß es sich um statistisch gesicherte Mittelwerte handelt. Diese Bezugsgröße erscheint als Vergleichsbasis verwertbar, da das HT-Tropfenvolumen, prozentual auf das Körpergewicht $(=100 \%)$ bezogen, während der gesamten Dauer der Larvalentwicklung gleichbleibt. Tab. 1 zeigt, daß das Tropfenvolumen ca. $6 \%$ des Körpergewichtes beträgt-eine Ausnahme machen nur ältere apter determinierte Larven (im folgenden kurz aptere bzw. auch alate Larven genannt) im dritten Stadium. Ihr Kottropfen- 
volumen liegt ca. $1 / 3$ höher. Da diese Tiere sich kurze Zeit später häuteten, sei hieran gezeigt, daß unsere Bezugsgröße « HT-Tropfen » unmittelbar vor der Ekdysis nicht mehr angewandt werden sollte.

TAB. 1. - Prozentualer Bezug des Kottropfenvolumens auf das Körpergewicht der Aphidenlarven in Abhängigkeit vom Lebensalter (Körpergewicht $=100 \%$ ).

TABL. 1. - Rapport en pourcentage du volume des gouttes d'excrétat au poids corporel des larves $d^{\prime}$ 'Aphidiens en fonction de l'âge (poids corporel $=100 \%$ ).

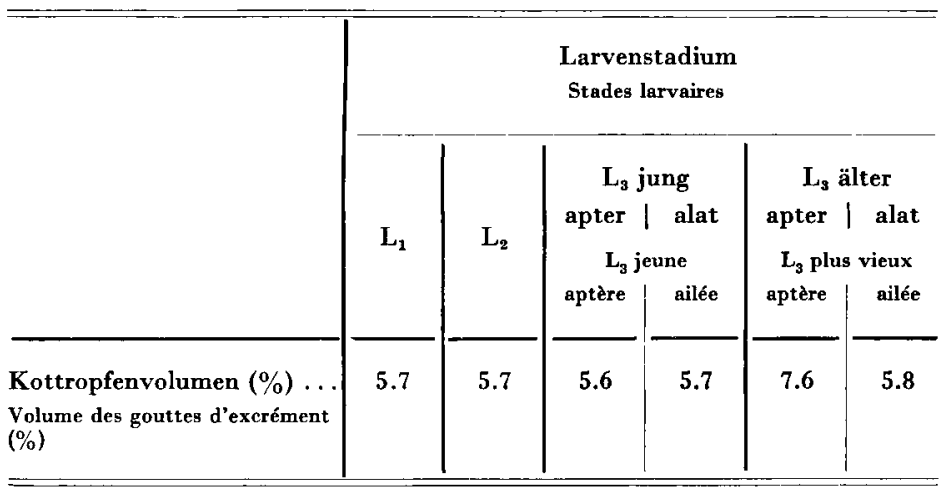

\section{3. - ERGEBNISSE}

Zuerst sei die HT-Analyse von Aphidenlarven an der Standarddiät nach Mittler et al. (1970), modifiziert nach Kunkel (1977), dargestellt (Abb. I). Man sieht, daß der N-Gehalt pro $1 \mu \mathrm{g}$ KH während aller Larvenstadien trotz gleichbleibender Nährlösung schwankt (bis zu $280 \%$ !) und daß alate Morphen mehr N (im Mittel $43 \%$ ) als ihre gleich ernährten apteren Geschwister exkretieren.

In Tab. 2 sind die durchschnittlichen Substanzmengen im HT der einzelnen Larvenstadien und Morphen angegeben. Von Stadium zu Stadium steigt der Gehalt aller hier untersuchten Inhaltsstoffe an. Dabei wird auch gezeigt, daß im HT alater Morphen von L3 und L4 mehr AS jedoch eigenartigerweise weniger Keto- sowie Aldohexosen enthalten waren. Diese beiden Kohlenhydratgruppen sind im HT mengenmäßig im Verhältnis $1: 4$ vorhanden, in der Nährlösung (Saccharose) dagegen im Verhältnis $1: 1$.

Ein weiterer, die HT-Zusammensetzung beeinflussender Faktor ist selbstverständlich die Zusammenstellung der Nährlösung (Tab. 3). Wie man sieht, vergrößert sich der HT-AS-Gehalt bei Verdoppelung der AS in der Nährlösung außerordentlich stark, die $\mathrm{KH}$ verhalten sich jedoch gegensinnig, sieht man vom ersten, sicherlich pathologischen Wert ab. Von Diättyp zu Diättyp sind die $\mathrm{KH} \mathrm{um} \mathrm{ca.} 20 \%$ verringert. Berechnet man hierzu wiederum den Gehalt von AS/1 $\mu \mathrm{g} \mathrm{KH,} \mathrm{erhält} \mathrm{man} \mathrm{die} \mathrm{Darstellung} \mathrm{der} \mathrm{Abb.} 2$. 


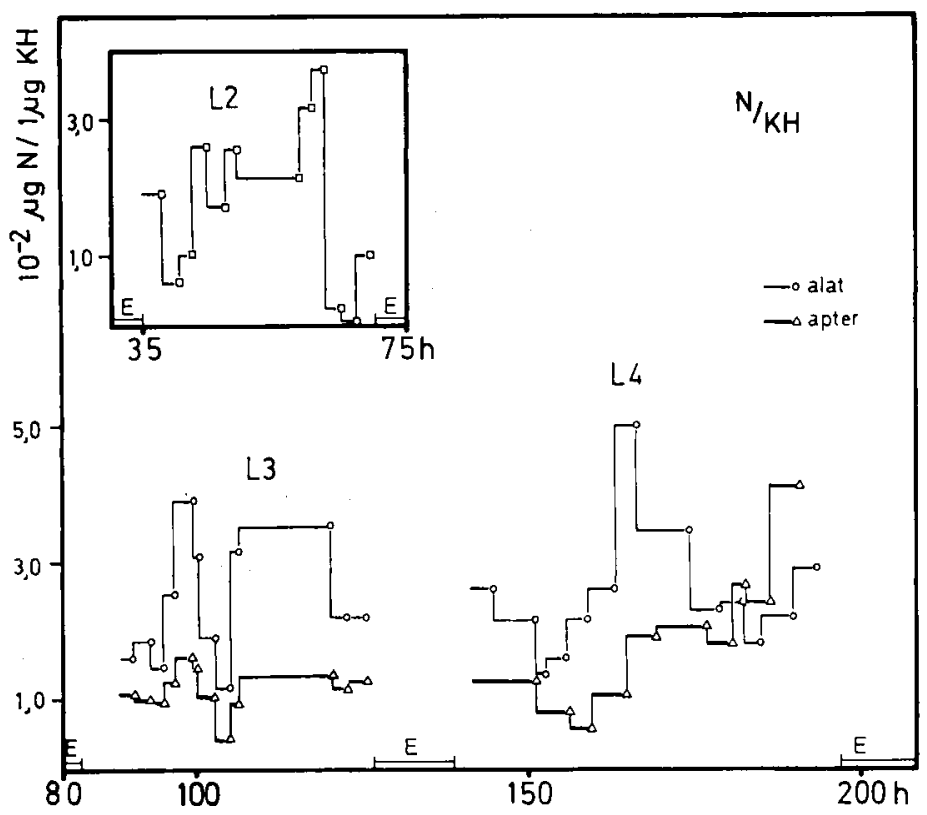

AвB. 1. - Stickstoffgehalt bezogen auf $1 \mu \mathrm{g}$ Kohlenhydrate im Honigtau von Aphidenlarven in Abhängigkeit vom Lebensalter in Stunden.

$\mathbf{L}_{2}, \mathbf{L}_{3}, \mathbf{L}_{4}$ bedeutet Larven im 2., 3. und 4. Stadium; apter = apteriform, alat = alatiform; $\mathbf{E}=\mathbf{E c d y s i s . ~}$ -O Zeitraum, in dem die analysierte Honigtauprobe gesammelt wurde.

FIG. 1. - Teneur en azote, rapportée à $1 \mu g$ d'hydrate de carbone, du miellat des larves d'Aphidiens en relation avec l'âge exprimé en heures.

$\mathrm{L}_{2}, \mathrm{~L}_{3}, \mathrm{I}_{4}$ signifient larves du $2^{\mathrm{e}}, \quad 3^{\mathrm{e}}$ et $4^{\mathrm{e}}$ stades; apter $=$ forme aptère; alat $=$ forme ailée; $\mathrm{E}=$ ecdysis; $-\mathrm{O}=$ espace dans lequel les échantillons ont été prélevés.

Eine lineare Abhängigkeit zwischen AS im Nahrungsangebot und denjenigen im HT besteht folglich nicht. Vielmehr verläuft die Kurve exponentiell. Die Werte der HT-Analysen, die von an der Pflanze saugenden Aphiden stammen, entsprechen denjenigen einer 1 bis 1.5 fachen AS-Diät, der ASGehalt des HT bezogen auf seinen KH-Inhalt schwankt dabei um den Faktor 3. HT-Analysen von Aphiden, die ausschließlich an der Pflanze parasitieren, sind in Abb. 3 dargestellt. Der KH-Gehalt fällt während ca. 100 Stunden nach etwa 60 Stunden auf ein Minimum und steigt danach wieder auf relativ hohe Werte an. In anderem Rhythmus ändert sich der AS-Gehalt : ca. alle 40 Stunden werden minimale Werte (hier 8, 44 und 80 Stunden nach Versuchsbeginn) erreicht. AS und KH sind im HT an der Pflanze saugender Aphiden auch nicht mengenmäßig korreliert sondern unterliegen eigenen Schwankungen. Wie stark sich das ausdrücken kann, zeigt exemplarisch Abb. 4. Innerhalb von 8 Stunden halbierte sich der N-Gehalt, wohingegen der KH-Anteil um den Faktor 4 von $2 \mu \mathrm{g}$ auf $8 \mu \mathrm{g}$ anstieg. 


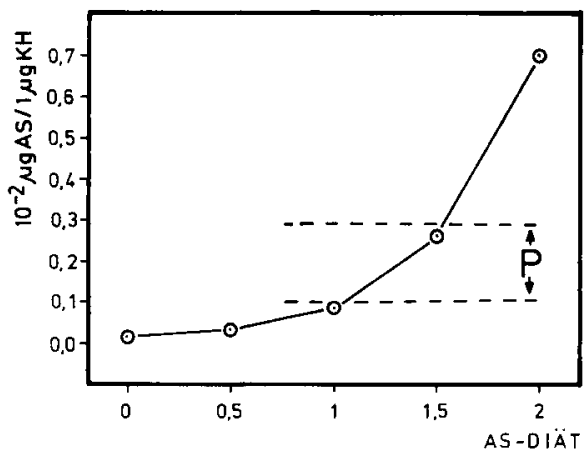

Aвв. 2. - Exponentieller Anstieg des Aminosäurengehaltes (AS), bezogen auf $1 \mu g$ Kohlenhydrate (KH), bei linear zunehmendem Diätaminosäurengehalt im Honigtau von Aphiden an den entsprechenden Nährlösungen. Kotanalysen von Aphiden an der Pflanze liegen im Bereich von « $\mathbf{P}$ ».

Fic. 2. - Croissance exponentielle de la teneur en acides aminés (AS), rapportée à $1 \mu \mathrm{g}$ d'hydrate de carbone $(K H)$, du miellat d'Aphidiens, lorsque la teneur en acides aminés du régime croit linéairement. Les analyses des excréments des Aphidiens nourris sur la plante se situent dans le domaine « $\mathbf{P}$ ».

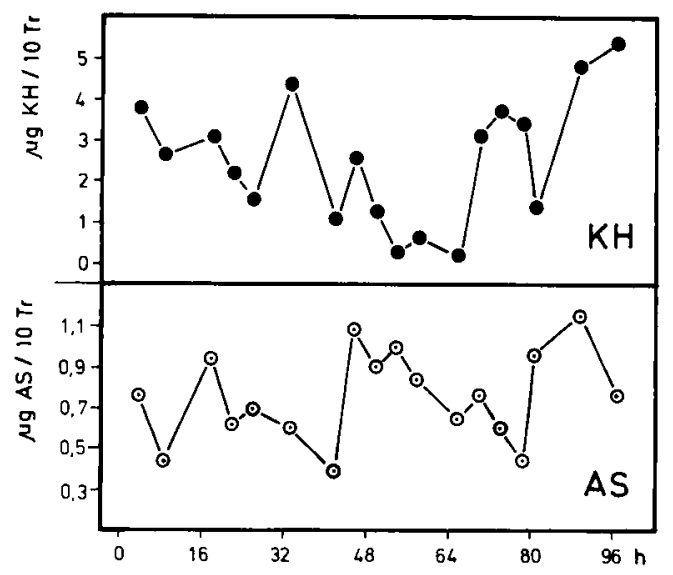

Aвв. 3. - Kohlenhydrat (KH) -und Aminosäurengehalt (AS) pro 10 Honigtautropfen (10 Tr) von Aphiden ( $L_{4}$, apter und Imagines) an der Pflanze im zeitlichen Verlauf.

Fig. 3. - Teneur en hydrates de carbone ( $K H)$ et en acides aminés ( $A S)$ de 10 gouttes de miellat (10 $\mathrm{Tr}$ ) d'Aphidiens ( $L_{4}$, forme aptère et imagos) nourris sur la plante en fonction du temps. 
TAB. 2. - Durchschnittliche Substanzmengen im Honigtau einzelner Larvenstadien und Morphen. Die Zahlenangaben sind Mittelwerte in $\mu \mathrm{g} / 10$ Tropfen \pm Vertrauensbereich $(P<5 \%)$. $=132$.

TABL. 2. - Quantités moyennes de substance dans le miellat des divers stades larvaires et des diverses formes. Les données chiffrées sont des moyennes en $\mathrm{ug} / 10$ gouttes \pm domaine de confiance $(\mathrm{P}<5 \%) . \mathrm{n}=132$.

\begin{tabular}{|c|c|c|c|}
\hline $\begin{array}{l}\text { L-Stadium }+ \text { Morphe } \\
\text { Stades larvaires et formes }\end{array}$ & $\begin{array}{l}\text { Aminosäuren } \\
\text { acides aminés }\end{array}$ & $\begin{array}{c}\text { Ketohexosen } \\
\text { Cétohexoses }\end{array}$ & $\begin{array}{c}\text { Keto- und } \\
\text { Aldohexosen } \\
\text { Céto- et aldohexoses }\end{array}$ \\
\hline $\mathbf{L}_{1}$ & 0.51 & 1.22 & 3.59 \\
\hline $\mathrm{L}_{2}$ & $0,80 \pm 0.3$ & $2.12 \pm 0.36$ & $7.88 \pm 1.21$ \\
\hline $\begin{array}{r}\mathbf{L}_{3} \text { apter } \\
\text { aptère }\end{array}$ & $1.13 \pm 0.3$ & $3.83 \pm 0.26$ & $17.81 \pm 0.94$ \\
\hline $\begin{array}{l}\text { alat } \\
\text { ailée }\end{array}$ & $1.69 \pm 0.5$ & $3.16 \pm 0.36$ & $12.44 \pm 1.22$ \\
\hline $\begin{array}{r}\mathrm{L}_{4} \text { apter } \\
\text { aptère }\end{array}$ & $3.44 \pm 1.2$ & $5.90 \pm 0.55$ & $33.91+3.82$ \\
\hline $\begin{array}{l}\text { alat } \\
\text { ailée }\end{array}$ & $4.66 \pm 1.3$ & $4.73 \pm 0.45$ & $32.21 \pm 2.66$ \\
\hline
\end{tabular}

Läßt man die Pflanze welken, verändert sich zeitabhängig die HT-Zusammensetzung sehr stark. Tab. 4 zeigt diesen Effekt. Mit zunehmendem Turgorverlust steigt der AS-Gehalt um $245 \%$ an, dabei allein um $77 \%$ im Zeitraum zwischen 20 und 8 Stunden vor völligem Erschlaffen des Blattes. Erst ca. 4. Stunden nach dem KH-Gehalt verringern sich die AS um ca. 2/3 ihres Gehaltes. Erstaunlich ist, daß schon 12 Stunden vor dem endgültigen Welken des Blattes KH nur noch in Spuren nachweisbar waren, nachdem sich ihr Gehalt in den 24. Stunden davor verfünfzehnfacht hatte. Zu dieser Zeit dürften die Aphiden wohl, wenn man sie nicht daran hindert, abwandern, und, wenn möglich, einen anderen Wirt aufsuchen. Auch unter der Bedingung des « latenten Wassermangels " sind beide untersuchten Substanzgruppen nicht korreliert und beeinflussen die HT-Zusammensetzung außerordentlich stark.

\section{4. - DISKUSSION}

Die von Aphiden an der holidischen Diät gewonnenen Daten zeigen, daß die HT-Zusammensetzung hinsichtlich der hier untersuchten Stoffklassen nicht konstant ist, obwohl die Nährlösung in ihrer Zusammensetzung während des Experimentes gleich bleibt. Der HT ändert sich vielmehr in Abhängigkeit vom 
Larvenstadium, vom Ablauf der ontogenetischen Entwicklung innerhalb der einzelnen Stadien und vom anteilmäßigen Auftreten beider Morphen in der Kolonie. Diese HT-Änderungen werden auf den sich ändernden Nährstoffbedarf der Aphiden im Laufe ihrer Ontogenie zurückgeführt (Hentel und Kunkel, 1976). Stoffwechsel-Bilanzgleichungen (KunKel und Hertel, 1975) zeigen sogar, daß die für beide Morphen nachgewiesene, unterschiedliche Nahrungsnutzung ein gutes Bild für die Anpassung der Tiere an ihren Lebensraum liefert : aptere Individuen als seßhafte, bei optimaler Nahrung ihren Wirt mit Nachkommen überschwemmende Parasiten benötigen viel AS (weshalb sie weniger im HT abgeben werden); alate Morphen hingegegen benötigen mehr KH zur Reservestoffsynthese für den Energiebedarf bei der Migration.

TAB. 3. - Durchschnittlicher prozentualer Aminosäuren- und Kohlenhydratgehalt im Honigtau von Aphidenlarven ( $L_{3}$, apter) in Abhängigkeit vom Diättyp. Die Werte der 1 AS-Diät sind $=100 \%$ gesetzt. Die 0 ASDiät enthält keine AS, die 0.5 AS-Diät die halbe Menge der 1 AS-Diät, 1.5 und 2 AS-Diät dem entsprechend.

TABL. 3. - Teneur moyenne, en pourcentage, en acides aminés et en hydrates de carbone du miellat des larves d'Aphidiens ( $L_{3}$, forme aptère) en fonction du régime. On attribue la valeur $100 \%$ au régime 1 AS. Le régime 0 AS ne renferme aucun acide aminé; le régime 0,5 AS contient moitié moins d'acides aminés que le régime $1 \mathrm{AS}$; les régimes $1,5 \mathrm{AS}$ et 2 AS respectivement 1,5 et 2 fois plus.

\begin{tabular}{|c|c|c|}
\hline \multirow{2}{*}{$\begin{array}{c}\text { Diättyp } \\
\text { Type de régime }\end{array}$} & \multicolumn{2}{|c|}{$\begin{array}{l}\text { Gehalt im Honigtau an } \\
\text { Teneur dans le miellat en }\end{array}$} \\
\hline & $\begin{array}{l}\text { Aminosäuren } \\
\text { acides aminés }\end{array}$ & $\begin{array}{c}\text { Kohlenhydraten } \\
\text { Hydrates } \\
\text { de carbone }\end{array}$ \\
\hline 0 AS & 14 & 107 \\
\hline $0.5 \mathrm{AS}$ & 45 & 120 \\
\hline 1 AS & 100 & 100 \\
\hline $1.5 \mathrm{AS}$ & 314 & 85 \\
\hline $2 \quad \mathrm{AS}$ & 611 & 59 \\
\hline
\end{tabular}

Weitere Änderungen in der HT-Zusamensetzung sind, wie eigentlich zu erwarten, durch wechselndes Nahrungsangebot zu erhalten; als Beispiel dienen unsere unterschiedlichen Diättypen. Zusätzlich zu diesen, in der Physiologie der Tiere resultierenden Faktoren tragen all jene Einflüsse zur HT-Änderung bei, die vom pflanzlichen Organismus abzuleiten sind (tageszeitlich bedingte Konzentrationsschwankungen der Assimilatstoffe, Änderung der Wasserversorgung). Beide Faktoren bewirken derart starke Schwankungen in der HTZusammensetzung, wie sie bei holidischer Ernährung nie erhalten wurden. 


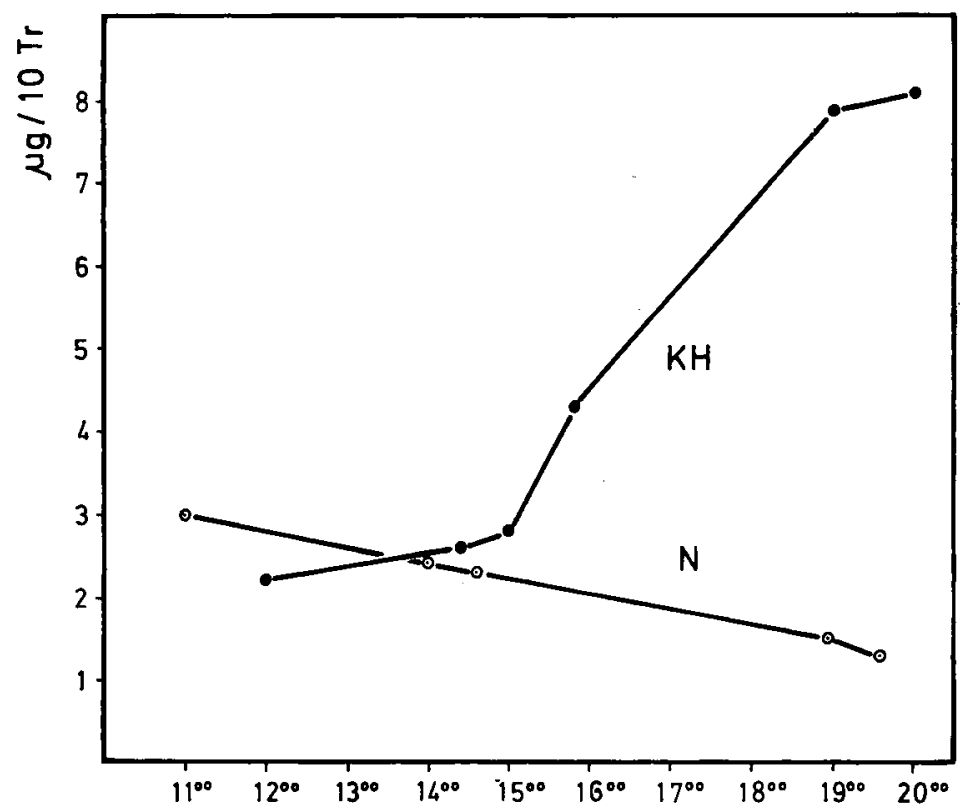

Aвв. 4. - Kohlenhydrat (KH) -und Stickstoffgehalt $(N)$ im Honigtau von Aphidenlarven ( $L_{3}$, apter) an der Pfanze in Abhängigkeit von der Tageszeit während eines Teils der Photophase.

Fig. 4. - Teneur en hydrates de carbone (KH) et en azote $(N)$ du miellat de larves d'Aphidiens ( $L_{3}$, forme aptère) nourries sur la plante en fonction de l'heure durant une partie de la photophase.

TAB. 4. - Abhängigkeit der Honigtauzusammensetzung (Imagines mit $L_{1}$ ) von der Turgeszenz des besaugten Blattes. Am Zeitpunkt 0 ist das Blatt völlig erschlafft. AS bzw. KH/Tr $=$ Aminosäuren bzw. Kohlenhydrate im Kottropfen.

'TABL. 4. - Relation entre la composition chimique du miellat (imagos et $L_{1}$ ) et la turgescence de la feuille sucée. Au point 0 la feuille est totalement flétrie. AS ou $\mathrm{KH} / \mathrm{Tr}=$ acides aminés ou hydrates de carbone présents dans la goutte de miellat.

\begin{tabular}{|c|c|c|c|c|c|c|c|}
\hline & $36-30 \underset{h}{\text { Std }}$ & $30-24$ & $24-20$ & $20-12$ & $12-8$ & $8-4$ & $4-0$ \\
\hline$\mu \mathrm{g} \mathrm{AS} / \mathrm{Tr} \ldots \ldots \ldots$ & 4.81 & 5.88 & 8.25 & 9.35 & 16.60 & 5.07 & 2.77 \\
\hline$\mu \mathrm{g} \mathrm{KH} / \mathrm{Tr} \ldots \ldots$ & 1.96 & 5.40 & 10.14 & 29.52 & $\begin{array}{l}\text { Spur } \\
\text { Traces }\end{array}$ & $\begin{array}{l}\text { Spur } \\
\text { Traces }\end{array}$ & 6.74 \\
\hline
\end{tabular}


Folgt aus einer großen Siedlungsdichte der Parasiten an der Pflanze eine Verschiebung in der Nährstoffbereitstellung (ev. können die dann beobachteten Kümmertriebe ein Anzeichen dafür sein) und ändert sich dabei die Morphenzusammensetzung der Kolonie, wird neben den bisher aufgezählten Faktoren auch noch die Anzahl der Individuen an der Pflanze Einfluß auf ihre HT-Zusammensetzung ausüben.

Eingegangen im Mai 1977.

Reçu pour publication en mai 1977.

\section{RÉSUMÉ}

Nous avons comparé la composition chimique en acides aminés et en hydrates de carbone du miellat des larves d'Aphidiens ayant reçu un régime naturel et un régime holidique. Dans des conditions standard la composition chimique du miellat dépend de l'heure à laquelle l'échantillon a été prélevé, de l'âge de l'insecte ainsi que de la composition de la colonie (rapport des deux formes aptère et ailée). Un autre facteur est constitué par la concentration variable en acides aminés et en hydrates de carbone du régime holidique et de la plante (changement dans la teneur en sève élaborée, variation de la quantité d'eau).

\section{LITERAT UR}

Boussingault M., 1872. - Sur une matière sucrée apparue sur les feuilles d'un tilleul. C.R Acad. Sci., Paris 74, 87-90.

Hertel R., 1971. - Untersuchungen zur Abgabefrequenz und Zusammensetzung des Honigtaus von Myzus persicae (Sulz.) (Aphidina), die mit einer künstlichen Diät ernährt wurden. Diplomarbeit, Bonn.

Hertel R., 1974 a. - Einfluß von ATP in einer holidischen Diät auf Myzus persicae (Sulz.) (Aphidina). Experientia 30, 775-776.

Hertel R., 1974 b. - Beitrag zur Physiologie und Ökologie der Ernährung von Myzus persicae (Sulz.) (Aphidina) unter besonderer Berücksichtigung ihrer beiden virginoparen Morphen in der Larvenphase. Dissertation, Bonn.

Hertel R., u. KunKel H., 1976. - Wechselnde Verwertung chemiseh definierter Nahrung durch Myzus persicae-Larven (Hemiptera : Aphididae). Zool. Jb. Physiol.,(B) 80, 259-266.

VoN HANDEL E., 1967. -- Determination of fructose and fructose yielding carbohydrates with cold anthrone. Anal. Biochem., 19, 193-194.

KunKeL H., 1977. - Membrane feeding systems in aphid research. In : K. F. Harris, K. Maramorosch, eds., Aphids as virus vectors. Academic Press London and New York.

Kunkel H., u. Hertel R., 1975. - Erstellung einer Stoffwechselbilanz für beide virginoparen Morphen von Myzus persicae (Sulz.). - Larven im dritten Stadium. Oecologia (Berl.) 18, 23-35.

Kunkel H., u. Hertel R., 1976. - Kotabgabe von holidisch ernährten Myzus persicae Larven (Aphidina, Homoptera) bei unterschiedlichen Experimentalbedingungen. Ent. exp. \& appl., 19, 82-95.

Mittler T. E., u. DadD R. H., 1962. - Artificial feeding and rearing of the aphid, Myzus persicae (Sulz.), on a completely defined synthetic diet. Nature, Lond., 195, 404.

Mittler T. E., Tsitsipis J. A., KleinJahn J. E., 1970. - Utilization of dehydroascorbic acid and some related compounds by the aphid Myzus persicae feeding on an improved diet. J. Insect Physiol., 16, 2315-2326.

Rosen H., 1957. - A modified colorimetric analysis for amino acids. Arch. Biochem. Biophys., 67, 10-15. 